\title{
Components of the Patient-Centered Medical Home Associated with Perceived Access to Primary Care
}

'VA Puget Sound Health Care System Health Services Research \& Development, Seattle, WA, USA; ${ }^{2}$ Department of Medicine, University of Washington, Seattle, WA, USA; ${ }^{3}$ King County Department of Community and Human Services, Seattle, WA, USA.

BACKGROUND: Following implementation of the patientcentered medical home (PCMH) within the Department of Veterans Affairs (VA), access to primary care improved. However, understanding of how this occurred is lacking. OBJECTIVE: To examine the association between organizational aspects of the PCMH model and access-related initiatives with patient perception of access to urgent, same-day, and routine care within the VA.

DESIGN: Cross-sectional

PARTICIPANTS: Veterans who responded to the annual Survey of Healthcare Experiences of Patients in $2016(N=$ 241,122 patients) and primary staff who responded to VA National Primary Care Provider and Staff Survey $(N=$ 4815 staff).

MAIN MEASURES: Three outcomes of perception of access: percentage of patients responding in the highest category for same-day care (waiting $\leq 1$ day), urgent care (always receiving care when needed), and routine care (always receiving checkups when desired). Predictors were staff-level report of access-related initiatives and organizational factors in the clinic. We used generalized estimating equations to model associations, adjusting for characteristics of patients and their respective clinics. KEY RESULTS: Access was significantly better in clinics where staff reviewed performance reports $(+0.9 \%$ in the highest perception of access for urgent care, $P<0.01 ;+$ $1.2 \%$ for routine care, $P<0.001$ ), leadership was supportive of the $\mathrm{PCMH}(+1.6 \%$ for urgent care, $P<0.01)$, and initiatives to improve access included open access (+ $0.8 \%$ to $+1.7 \%$ across all outcomes, $P<0.01)$ and telehealth visits $(+1.2 \%$ to $+1.4 \%, P<0.001)$. Perceived access was worse in clinics with moderate staff burnout $(-1.1 \%$ to $-1.4 \%, P<0.001)$, primary care provider turnover during the past year $(-1.0 \%$ to $-1.6 \%, P<0.001)$, or medical support assistant turnover in the past year ($0.9 \%$ to $-1.4 \%, P<0.001)$.

CONCLUSIONS: Perception of access was strongly associated with identifiable organizational factors and accessrelated initiatives within VA primary care clinics that could be adopted by other health systems.

Electronic supplementary material The online version of this article (https://doi.org/10.1007/s11606-020-05668-7) contains supplementary material, which is available to authorized users.

Received August 7, 2019

Revised November 27, 2019

Accepted January 12, 2020

Published online January 28, 2020
J Gen Intern Med 35(6): 1736-42 DOI: $10.1007 / \mathrm{s} 11606-020-05668-7$

(C) Society of General Internal Medicine (This is a U.S. government work and not under copyright protection in the U.S.; foreign copyright protection may apply) 2020

\section{INTRODUCTION}

Better primary care access is associated with higher patient satisfaction, lower mortality, and improved medication adherence. ${ }^{1-3}$ However, not all strategies to increase access are equally effective and may have unintended consequences when interacting. When implemented in isolation, some strategies such as open (i.e., advanced) access may lead to improved wait times without clear benefit for health outcomes or utilization. ${ }^{4,5}$ Alternatively, new access initiatives may have unanticipated conflicts when added to existing strategies - for example, implementing telemedicine visits might expand scheduling capacity but create unforeseen barriers for patients with limited digital literacy. While administrative measures are commonly used to define access, ${ }^{3}$ perceived access is equally valid and more relevant to the patient's experience. ${ }^{6,}{ }^{7}$ For health systems seeking to improve access, an important first step is examining the individual contributions of strategies that might influence patients' perception of access.

In 2010, the Veterans Health Administration of the Department of Veterans Affairs (VA) implemented a version of the patient-centered medical home (PCMH), the Patient Aligned Care Team (PACT) initiative, across all primary care clinics. ${ }^{8}$ Specific access-related initiatives of PACT included increased staffing and expanding virtual encounters through telephone visits, secure messages, and telehealth video. ${ }^{9}$ Open access (defined as a focus on enhanced continuity with expanded capacity for visits) was also implemented, with local PACT control over specific open access techniques such as designating same-day or urgent slots in continuity team schedules. 9,10 While access continued to improve within the VA over the past decade despite increasing demand, ${ }^{11,}{ }^{12}$ which initiatives and factors of PACT were most influential for access is less understood. We sought to assess the association of access-related initiatives and factors of the PCMH with patients' perceived access to same-day, urgent, and routine care using data from over 700 primary care clinics. 


\section{METHODS}

\section{Study Design}

We conducted a cross-sectional study using 2016 VA data. Perception of access was measured using questions from the Consumer Assessment of Healthcare Providers and Systems (CAHPS) collected from a nationally administered survey, the VA Survey of Healthcare Experiences of Patients (SHEP CAHPS-PCMH version). Patient responses were linked to their designated primary care clinic and averaged by clinic for each access outcome (same-day, urgent, and routine care). To determine components of the PCMH model associated with access, we used staff responses on the availability of clinic access-related initiatives and organizational factors using the VA National Primary Care Provider and Staff Survey. Clinic-level averages of access outcomes were linked to staff survey responses for staff-level adjusted comparisons. Generalized estimating equations modeled the association between predictors and outcomes. This study was part of the VA's evaluation for the PACT model and was considered quality improvement rather than research, and thus not subject to institutional review board approval or waiver.

\section{Patient Perception of Access Outcomes}

Patient perception of access was assessed by responses to the SHEP CAHPS in 2016 among patients with an assigned primary care provider $(n=241,122$, Supplemental Table e1). The SHEP CAHPS is routinely administered by mail to a random sample of outpatients, stratified by division, with encounters in the past month. ${ }^{13}$ The 2016 overall response rate was $41.2 \%$. The SHEP CAHPS incorporates sampling weights to adjust for proportionate population representation and survey non-response by age, gender, and site size. The access scales have acceptable internal consistency (Cronbach's $\alpha=0.74$ ) and practice-level reliability $(0.87) .{ }^{14}$ Access-related survey questions are scaled ordinally from 1 to 4 or from 1 to 5 , with higher scores indicating better access perception. For models, we created a binary variable using either the top or top-two scale responses, i.e., "top-box" or "toptwo." Three questions were used as outcomes. First, we considered same-day access as responses of "same day" or "1 day" to the question, "How many days did you have to wait for an appointment when you needed care right away?" We considered urgent care access as responses of "always" to the question, "For care you needed right away, how often did you get an appointment as soon as you needed?" We considered routine care access as responses of "always" to the question, "When you made an appointment for a check-up or routine care with this provider, how often did you get an appointment as soon as you needed?" We calculated each clinic's percentage of patients selecting the top-box response (or top-two responses for same-day care) for the three outcomes. Routine care, selected as non-urgent requests, could have more difficulty with timely access and was used as the outcome to describe the highest and lowest performing clinic deciles for descriptive analyses.

\section{The PACT Model and Access-Related Predictors}

PACT is an interdisciplinary PCMH model focused on teambased care, expanded access, chronic disease management, care coordination, and patient centeredness. Within PACT, patients are assigned to "teamlets" with a primary care provider (PCP) (physician, nurse practitioner, or physician's assistant), registered nurse ( $\mathrm{RN})$ care manager, clinical associate (licensed practical (LP) nurses or vocational nurses (VNs)), and clerical medical support assistant (MSA). Teamlets are part of larger PACT teams, which includes integrated behavioral health staff, social workers, and clinical pharmacists.

Predictors were from PACT staff responses to the 2016 VA National Primary Care Provider and Staff Survey $(n=4815$ staff within 713 clinics) on ratios of staff support for PCPs, burnout, turnover, clinic characteristics, and use of accessrelated initiatives. The survey was anonymously administered through e-mail invitations sent at the regional level to primary care staff. Response rates to the staff survey were estimated at $18 \%$ for 2016, based on administrative records for 30,182 staff within 8256 PACT teamlets. Clinic assignment and demographics were self-identified by participating staff (Supplemental Table e1). Four core PACT roles were assessed: PCP, RN, LP/VN, and MSA. Two groups of predictors were included from the survey: 11 questions on clinic organizational factors and 11 questions on the presence of access-related initiatives used at clinics.

We used multiple imputations to account for item-level nonresponse for organizational factors on the PACT survey using SAS Enterprise Guide 7.4 (PROC MI command). Due to categorical variables, we used fully conditional specification (FCS) to impute missing data. ${ }^{15}$ This method is better suited for datasets that contain both categorical and continuous variables and uses a multivariate imputation model on a variableby-variable basis. In total, 50 imputed datasets were created with a burn-in of 10 iterations (i.e., iterations for convergence to a distribution before imputation). ${ }^{16}$ Out of 11 staff survey questions in this category, 4 had high levels of missingness: written role descriptions for staff (18.7\%), supportive leadership structure $(16.3 \%)$, team review of performance reports $(15.1 \%)$, and presence of a fully staffed PACT $(8.2 \%)$. The remaining 7 items had missingness $<5 \%$. For each predictor to be imputed, all remaining variables (including all accessrelated predictors, auxiliary variables to produce the most accurate estimation, outcomes, and covariates) were included in a logistic regression model. One clinic was excluded from the access-related initiative models due to unit non-response. With this exception, no items were missing for the accessrelated initiative responses; therefore, no imputation was used.

\section{Data Sources and Covariates}

Administrative data for covariates were extracted from the VA Corporate Data Warehouse (CDW). ${ }^{17}$ Patient and clinic char- 
acteristics from 2016 were included a priori in adjusted models. Since patient-level demographics could not be linked directly to the anonymous staff-level survey responses (identifiable only at the clinic level), patient demographics were averaged by clinic to create covariates. These included patient age, sex as a binary indicator if the clinic had $\geq 10 \%$ of female patients, Elixhauser comorbidity score from ICD-10 principle diagnoses, ${ }^{18}$ and clinic community- or hospital-based affiliation.

\section{Statistical Analysis}

Unadjusted comparison of characteristics of clinical scoring within the highest and lowest deciles of routine care access for descriptive analyses was done using $t$ tests assuming unequal variance. For adjusted comparisons, each set of predictors (a set of 11 access-related initiative variables and a separate set of 11 organizational factor variables) was modeled simultaneously for each outcome, for a total of six models. Models were adjusted for patient and clinic characteristics. As each outcome was a percentage of patient responses in the top-box/top-two boxes at the clinic level and followed a normal distribution, we used generalized estimating equations with an identity link for the main models. We used independent covariance structures to accommodate correlation of responses within clinics. The unit of analysis in the adjusted analyses was at the staff level ( $N=4815)$, as this was the most granular data between patient and staff responses. Additionally, condensing the staff responses further to the clinic-level unit of analysis would have resulted in data loss as only 713 clinics were involved.

Secondary analyses included outcome stratification by staff role for five subgroups (PCP, RN, LPN/LVN, MSA, and LPN/ LVN + MSA). Standard errors for coefficient estimates were heteroskedastic robust. All hypothesis testing was two sided with an alpha value of 0.05 . Analyses were performed using SAS Enterprise Guide, version 7.4 (SAS Institute Inc., Cary, NC).

\section{RESULTS}

Top-performing clinics with respect to access to routine care had, on average, older and more male patients, with lower staff turnover or burnout and greater use of open access and virtual care than low-performing clinics (Table 1). Patients seen at top-performing clinics for access to routine care also reported significantly better access to same-day $(51.5 \%)$ and urgent $(66.2 \%)$ care compared to low-performing clinics $(P<0.001)$. On average for all clinics, $58.9 \%$ of patients reported that they always received urgent care when needed, $38.2 \%$ of patients reported that they waited a day or less, and $58.9 \%$ of patients reported receiving routine checkups as soon as needed. Across all clinics, almost two thirds of staff reported that at least one team member had left the team within the past year. Of those responding to the staff survey, 1506
(31.3\%) were PCPs, 1483 (30.8\%) RNs, 1133 (23.5\%) LV/ LPNs, and 693 (14.4\%) MSAs.

In adjusted models (Table 2), the organizational factors that were significantly associated with worse perception of all three types of access included the following: staff burnout $(-1.1 \%$ to $-1.4 \%$ of patients in the top box of access, $P<0.001)$; loss or turnover of the PCP $(-1.0 \%$ to $-1.6 \%, P<0.001)$, RN ($0.6 \%$ to $-1.0 \%, P \leq 0.03)$, or MSA $(-0.9 \%$ to $-1.4 \%$, $P<0.001)$ on a team within the past year; and use of written role descriptions $(-1.0 \%$ to $-1.1 \%, P \leq 0.03)$. Reporting a fully staffed PACT team was associated with $1.2 \%$ worse perception of access for both same-day care and urgent care $(P<0.001)$. Use of daily huddles was associated with $1.0 \%$ worse perception of access for routine care $(P<0.001)$. Better perceived access, conversely, was associated with leadership supportive of the PCMH $(+1.6 \%$ for urgent care, $P<0.01)$ and with regular performance report review $(+0.9 \%$ for urgent care, $P=0.01 ;+1.2 \%$ for routine care, $P<0.001)$. Loss of the clinical associate on the respondent's team was moderately associated with $0.7 \%$ better access for same-day care $(P=$ $0.04)$. Whether work was matched to training was unrelated to access.

For staff report of use of access-related initiatives, visits to the clinical pharmacist were associated with worse perception of access on all three outcomes $(-0.7 \%$ to $-1.3 \%$ of patients in top box of access, $P \leq 0.02$ ). Better perception of all access outcomes was associated with open access $(+0.8 \%$ to $+1.7 \%$, $P \leq 0.01)$ and availability of virtual/telehealth visits $(+1.2 \%$ to $+1.4 \%, P<0.001)$. Use of secure electronic messaging was associated with $1.4 \%$ better access for urgent care $(P=0.02)$. Waiting to schedule appointments until within 90 days of a planned visit (i.e., recall scheduling) was associated with $0.8 \%$ better perception of access for urgent care $(P=0.03)$ and $0.9 \%$ better perception of access for routine care $(P<0.01)$. None of the remaining initiatives were associated with differences in access perception.

In secondary analysis by staff respondent role (e.g., PCP or $\mathrm{RN})$, both the direction and percentage of differences in perceived access were qualitatively similar to that of the main models (Supplemental Table e2). Of note, burnout reported by staff across all roles was associated with worse perception of access for all three outcomes, except for the MSA role for routine care access: access was perceived as $1.5 \%$ better if MSAs reported burnout $(P=0.02)$. Open access was the only predictor significantly associated with a difference in perception of an access outcome across all staff roles $(+1.3 \%$ to + $2.5 \%$ better perception of urgent care access, $P \leq 0.03$ ).

\section{DISCUSSION}

In this cross-sectional study of over 241,000 patients receiving primary care at 713 clinics in 2016, we observed several access initiatives and organizational factors associated with a patient's perception of how readily they could access primary 
Table 1 Clinic and Patient Characteristics at Clinic-Level Overall and by Top and Bottom 10\% of Clinics by Perceived Access to Routine Care, 2016

\begin{tabular}{|c|c|c|c|c|}
\hline & $\begin{array}{l}\text { All clinics, } n= \\
713\end{array}$ & $\begin{array}{l}\text { Top } 10 \% \text { of clinics, } n= \\
71\end{array}$ & $\begin{array}{l}\text { Bottom } 10 \% \text { of clinics, } n= \\
71\end{array}$ & $\begin{array}{l}P \text { value (top vs. bottom } \\
\text { clinics) }\end{array}$ \\
\hline $\begin{array}{l}\text { Age of patients in years, mean } \\
\text { (SD) }\end{array}$ & $62.8(3.9)$ & $65.1(2.8)$ & $59.2(4.1)$ & $<0.001$ \\
\hline Female patients, $\%$ & 7.2 & 4.8 & 11.0 & $<0.001$ \\
\hline Elixhauser of patients, mean (SD) & $1.5(0.3)$ & $1.4(0.3)$ & $1.4(0.3)$ & 0.42 \\
\hline $\mathrm{CBOC}, \%$ & 78.0 & 94.4 & 80.3 & $<0.01$ \\
\hline Top-box urgent care, $\%$ & 49.7 & 66.2 & 33.2 & $<0.001$ \\
\hline Top-box same-day care, \% & 38.2 & 51.5 & 27.3 & $<0.001$ \\
\hline Top-box routine care, $\%$ & 58.9 & 76.8 & 40.0 & $<0.001$ \\
\hline $\begin{array}{l}\text { Patient panel per PCP, mean } \\
(\mathrm{SD})^{*}\end{array}$ & $936.2(224.1)$ & $931.7(227.6)$ & $998.1(226.4)$ & 0.09 \\
\hline Organizational factors ( $\%$ present) & - & - & - & - \\
\hline Burnout reported by staff & 40.0 & 33.5 & 44.4 & 0.06 \\
\hline Any staff turnover in past year & 65.1 & 52.7 & 72.0 & $<0.01$ \\
\hline Written role descriptions for staff & 77.1 & 79.0 & 78.8 & 0.97 \\
\hline $\begin{array}{l}\text { Fully staffed with } \geq 3 \text { support } \\
\text { staff }\end{array}$ & 63.5 & 64.4 & 55.2 & 0.23 \\
\hline PACT daily huddles & 63.9 & 60.1 & 58.4 & 0.78 \\
\hline Work well matched to training & 59.9 & 58.3 & 57.7 & 0.92 \\
\hline $\begin{array}{l}\text { Team reviews performance } \\
\text { reports }\end{array}$ & 66.9 & 76.4 & 56.7 & $<0.01$ \\
\hline Leadership maintains PACT & 84.5 & 87.6 & 81.9 & 0.18 \\
\hline Access initiatives (\% present) & - & - & - & - \\
\hline Clinical pharmacy visits & 57.4 & 39.5 & 57.4 & $<0.01$ \\
\hline Telephone visits & 86.4 & 89.8 & 83.4 & 0.10 \\
\hline Nursing visits & 91.2 & 93.2 & 89.1 & 0.18 \\
\hline Patient group visits & 21.5 & 21.9 & 21.1 & 0.89 \\
\hline Virtual care (telehealth) visits & 51.0 & 65.2 & 40.3 & $<0.001$ \\
\hline Secure electronic messaging & 93.9 & 95.3 & 92.3 & 0.24 \\
\hline No-show reports & 49.7 & 52.0 & 47.3 & 0.39 \\
\hline Phone reminders for appointments & 66.8 & 59.9 & 58.5 & 0.80 \\
\hline Recall scheduling for future visits & 83.1 & 88.7 & 78.7 & 0.02 \\
\hline Carve-out slots to hold times & 35.7 & 35.1 & 31.4 & 0.50 \\
\hline Open access scheduling & 49.4 & 59.3 & 34.4 & $<0.001$ \\
\hline
\end{tabular}

Clinic-level descriptives shown here are similar to presentation by staff level $(N=4815)$

$P A C T=$ Patient Aligned Care Team, CBOC community-based outpatient

*Panel size of patients per primary care provider (PCP), for 710 clinics (70 clinics in bottom 10\%, 71 in top 10\% by routine access); 3 clinics were not reported due to inaccurate or missing panel size data

Table 2 Differences in Mean (SE) Percentages of Patients Reporting Higher Perceived Access Associated with the Presence of Organizational Factors and Access-Related Initiatives in Primary Care Clinics

\begin{tabular}{|c|c|c|c|c|c|c|}
\hline & Same-day care & $\boldsymbol{P}$ & Urgent care & $\boldsymbol{P}$ & Routine care & $\boldsymbol{P}$ \\
\hline \multicolumn{7}{|l|}{ Organizational factors } \\
\hline Staff moderate or higher burnout & $-1.3(0.3)$ & $<0.001$ & $-1.4(0.3)$ & $<0.001$ & $-1.1(0.2)$ & $<0.001$ \\
\hline In past year, PCP changed or left team & $-1.2(0.3)$ & $<0.001$ & $-1.6(0.3)$ & $<0.001$ & $-1.0(0.3)$ & $<0.001$ \\
\hline In past year, RN changed or left team & $-1.0(0.3)$ & $<0.01$ & $-0.7(0.3)$ & 0.03 & $-0.6(0.3)$ & 0.03 \\
\hline In past year, LVN/LPN changed or left team & $0.7(0.3)$ & 0.04 & $0.2(0.3)$ & 0.58 & $0.1(0.3)$ & 0.76 \\
\hline In past year, MSA changed or left team & $-1.4(0.3)$ & $<0.001$ & $-1.1(0.3)$ & $<0.001$ & $-0.9(0.3)$ & $<0.001$ \\
\hline Written role descriptions used for staff & $-1.0(0.4)$ & 0.03 & $-1.1(0.4)$ & 0.01 & $-1.0(0.4)$ & 0.01 \\
\hline Team staffed at a full support-to-provider ratio of $3: 1$ & $-1.2(0.3)$ & $<0.001$ & $-1.2(0.3)$ & $<0.001$ & $-0.4(0.3)$ & 0.09 \\
\hline Daily huddle at primary care clinic & $-0.2(0.3)$ & 0.59 & $-0.4(0.3)$ & 0.13 & $-1.0(0.2)$ & $<0.001$ \\
\hline Work well matched to training & $-0.2(0.3)$ & 0.42 & $-0.5(0.3)$ & 0.08 & $0.0(0.2)$ & 0.91 \\
\hline Team regularly reviews performance reports & $0.6(0.4)$ & 0.08 & $0.9(0.4)$ & 0.01 & $1.2(0.3)$ & $<0.001$ \\
\hline Leadership maintains medical home model & $0.8(0.5)$ & 0.09 & $1.6(0.5)$ & $<0.01$ & $0.8(0.4)$ & 0.08 \\
\hline \multicolumn{7}{|l|}{ Access-related initiatives } \\
\hline Clinical pharmacist visits & $-0.7(0.3)$ & 0.02 & $-1.2(0.3)$ & $<0.001$ & $-1.3(0.3)$ & $<0.001$ \\
\hline Telephone visits & $0.0(0.4)$ & 0.95 & $-0.1(0.4)$ & 0.81 & $-0.4(0.4)$ & 0.27 \\
\hline Nursing visits & $-0.6(0.5)$ & 0.23 & $0.2(0.5)$ & 0.66 & $0.7(0.4)$ & 0.09 \\
\hline Patient group visits & $-0.1(0.3)$ & 0.67 & $0.3(0.3)$ & 0.43 & $0.2(0.3)$ & 0.46 \\
\hline Virtual care (telehealth video) visits & $1.2(0.3)$ & $<0.001$ & $1.2(0.3)$ & $<0.001$ & $1.4(0.3)$ & $<0.001$ \\
\hline Secure electronic messaging & $0.0(0.6)$ & 0.95 & $1.4(0.6)$ & 0.02 & $0.9(0.5)$ & 0.07 \\
\hline No-show reports used by clinic & $0.3(0.3)$ & 0.28 & $-0.2(0.3)$ & 0.44 & $-0.3(0.3)$ & 0.23 \\
\hline Telephone reminders for appointments & $-0.2(0.3)$ & 0.62 & $-0.3(0.3)$ & 0.28 & $0.1(0.3)$ & 0.73 \\
\hline Future appointments scheduled $\leq 90$ days (recall) & $-0.4(0.4)$ & 0.24 & $0.8(0.4)$ & 0.03 & $0.9(0.3)$ & $<0.01$ \\
\hline Carve-out times hold same-day appointments & $0.1(0.3)$ & 0.83 & $-0.5(0.3)$ & 0.11 & $-0.3(0.2)$ & 0.19 \\
\hline Open access scheduling & $1.5(0.3)$ & $<0.001$ & $1.7(0.3)$ & $<0.001$ & $0.8(0.2)$ & $<0.01$ \\
\hline
\end{tabular}

PCP primary care provider, $R N$ registered nurse, LPN licensed practical nurse, LVN licensed vocational nurse, MSA medical support assistant 
care with the VA. The circumstances most highly associated with worse perception of access were loss of the PCP in the past year, followed by burnout of the responding staff member or loss of the MSA within the team. Use of open access was most strongly associated with better perceived access, followed by a supportive leadership structure.

The overall PCMH model has potential to improve access, primarily through increased capacity. ${ }^{19,} 20$ The PCMH requires reorganizing care from the traditional, physiciancentric model of care to an interdisciplinary design. ${ }^{21}$ This can potentially increase capacity by redistributing the PCP workload to other members of the team. Further capacity increases could be accrued through reducing staff burnout and lower resultant turnover following implementation of the PCMH. ${ }^{22,}{ }^{23}$ Finally, the potential greater efficiency of service delivery with the PCMH model reduces waste, also improving capacity. ${ }^{24}$ While the PCMH model may overall improve access, our findings offer further insights into specific components and their interactions.

PCMH components associated with worse perceived access were similar to those found in previous literature. PCP turnover has been shown to be associated with worse patient experience of care-including access - but the degree of diminishment is related to continuity of the relationship. ${ }^{23}$ PCMH implementation within the VA did slightly increase turnover of PCPs, but following implementation, average years of PCP experience remained high (on average, 9.4 years) and overall $\leq 4 \%$ of PCPs turned over quarterly after PACT implementation. ${ }^{25}$ Consistent with our findings, turnover and burnout are likely related in terms of association with worse access. Prior research has shown that higher burnout is correlated with a risk of turnover, and replacing team members leads to higher burnout among remaining staff. ${ }^{26}$ In contrast to findings about burnout among the other clinical staff, the observation that higher degrees of reported burnout among MSAs were associated with better perception of access may reflect the greater workload required to respond quickly to patient requests for appointments. ${ }^{27,} 28$

Open access was most strongly correlated with better patient perception of access. Staff reporting their clinic furnished open access could indicate staff perception of the clinic as "good" for access, in alignment with patient perception. In other health systems, open access implementation was also associated with improved wait times. ${ }^{4}$ At some clinics in the VA, implementation of open access preceded the PCMH initiative and its success was associated with leadership engagement and staffing resources as well as regular team review of related data - also consistent with our findings about supportive leadership. ${ }^{10,29}$ Continuity, though not explicitly measured, is closely related to many of these concepts. PCMH emphasis on preserving continuity with $\mathrm{PCPs} /$ teams while expanding capacity (i.e., open access principles) potentially has greater pay-offs for perceived access than expanding capacity at the expense of continuity, as manifested by worse perception of access with carve-out slots held for same-day appointments.

Several of our findings were counter to what we expected. First, patients perceived worse access when teams use written role descriptions. We speculate that clinics which enforce rigid roles and responsibilities may lack flexibility to promptly accommodate patient needs. ${ }^{30}$ Secondly, and more puzzling, worse perception of access was associated with staff reporting that their PACT teams met the recommended ratio of support staff to PCP. While geography was not evaluated in this study, this may be due to relationships between location, patient demand, and workforce recruitment.

Our study has several limitations. Our data were crosssectional, which precludes causal inferences; we were limited to 2016 given survey question changes in other years. Additionally, our data were derived from surveys. While our use of survey weights was designed to compensate for patient survey non-response, the low staff survey response introduces the possibility of selection bias. We employed rigorous methods for imputation of item-level missingness within the staff survey but could not account for overall non-response given imprecise data on respondents. Finally, as with any observational study, baseline demographic differences and other unmeasured confounders among clinics may have biased our findings despite extensive adjustment (e.g., higher proportions of younger and female patients who attended clinics with worse perceived access to routine care). ${ }^{31}$

Our study has implications for both healthcare policy and clinical practice. Our results, though representing small absolute differences in perceived access, assume greater magnitude when considering collective impacts and scaled across the VA, which provided care for 6 million patients in 2016. ${ }^{32}$ Improving access is a key strategic goal at the national healthcare level and within the Veterans Health Administration. ${ }^{33,} 34$ Additionally, there are direct relationships between access and patients retaining care within a healthcare system, ${ }^{35}$ which is important for systems like the VA with global budgets determined by real or projected use of services. This is of additional significance for the VA given expansions into community-based services to offset when access is objective or perceived to be limited (e.g., the MISSION Act of 2018). ${ }^{36}$ The components that influence access are also individually important, and the inter-relationships between elements should be considered by systems hoping to improve access to primary care. For example, improving access through maximizing continuity and reducing PCP turnover could lead to net savings through reduced patient utilization of high-cost services and fewer expenses for replacing PCPs. ${ }^{26}, 37,38$ Similarly, access for some populations may marginalize others - such as negative impacts on follow-up care for patients with chronic conditions from expanding open access. ${ }^{39}$ The interactions between elements require careful consideration prior to real-world application, but deliberate combinations, selectively implemented, hold promise. 
In summary, our study is among the first to investigate the degree to which individual elements within the PCMH affect patient perception of access across a large integrated health system. While we found low overall absolute change in perception of access associated with any one element, our study suggests specific PCMH components which could be leveraged by systems to improve patient perception of access.

Acknowledgements: Our thanks to the Office of Reporting, Analytics, Performance, Improvement, and Deployment (RAPID) within the Veterans Health Administration for access to the SHEP CAHPS data. Additional thanks go to the members of the VA Primary Care Analytics Team (PCAT) for insights. This work was undertaken as part of the national evaluation of PACT funded by the VA Office of Primary Care. Support for the primary author was from a VA HSR\&D Advanced Physician Fellowship. Funding agencies had no role in the study's design, conduct, or reporting. The views expressed are those of the authors and do not necessarily reflect the position of the affiliated institutions. This work was previously presented at the Society of General Internal Medicine annual conference (Denver, CO, April 1114, 2018).

Corresponding Author: Linnaea Schuttner, MD, MS; Department of Medicine University of Washington, Seattle, WA, USA (e-mail: linnaea. schuttner@va.gov).

\section{Compliance with Ethical Standards:}

Conflict of Interest: The authors declare that they do not have a conflict of interest.

\section{REFERENCES}

1. Meo N, Wong $\mathbf{E}$, Sun $\mathbf{H}$, et al. Elements of the Veterans Health Administration patient-centered medical home are associated with greater adherence to oral hypoglycemic agents in patients with diabetes. Popul Health Manag. 2018;21(2). doi:https://doi.org/10.1089/pop.2017. 0039

2. Prentice JC, Davies ML, Pizer SD. Which outpatient wait-time measures are related to patient satisfaction? Am J Med Qual. 2014;29(3):227235. doi:https://doi.org/10.1177/1062860613494750

3. Prentice JC, Pizer SD. Delayed access to health care and mortality. Health Serv Res. 2007;42(2):644-662. doi:https://doi.org/10.1111/j. 1475-6773.2006.00626.x

4. Rose K, Ross JS, Horwitz LI. Advanced access scheduling outcomes: A systematic review. Arch Intern Med. 2011;171(13):1150-1159. doi:https://doi.org/10.1001/archinternmed.2011.168

5. Degani N. Impact of advanced (open) access scheduling on patients with chronic diseases: an evidence-based analysis. Ont Health Technol Assess Ser. 2013;13(7):1-48.

6. Fortney JC, Burgess JF, Bosworth HB, Booth BM, Kaboli PJ. A reconceptualization of access for 21st century healthcare. $J$ Gen Intern Med. 2011;26(Suppl 2):639-647. doi:https://doi.org/10.1007/s11606011-1806-6

7. Kaboli PJ, Fihn SD. Waiting for care in Veterans Affairs Health care facilities and elsewhere. JAMA Netw Open. 2019;2(1):e187079. doi:https://doi.org/10.1001/jamanetworkopen.2018.7079

8. Rosland A-M, Nelson $\mathbf{K}$, Sun $\mathbf{H}$, et al. The patient-centered medical home in the Veterans Health Administration. Am J Manag Care. 2013;19(7):e263-272.

9. U.S. Department of Veterans Affairs. Patient Aligned Care Team (PACT) Handbook. May 2017. https://www.va.gov/VHAPUBLICATIONS/ ViewPublication.asp?pub_ID=2977. Accessed November 27, 2019.

10. True G, Butler AE, Lamparska BG, et al. Open access in the patientcentered medical home: lessons from the Veterans Health Administration. $J$ Gen Intern Med. 2013;28(4):539-545. doi:https://doi.org/10.1007/ s11606-012-2279-y

11. Wong ES, Rosland A-M, Fihn SD, Nelson KM. Patient-centered medical home implementation in the Veterans Health Administration and primary care use: differences by patient comorbidity burden. $J$ Gen Intern Med. 2016;31(12): 1467-1474. doi:https://doi.org/10.1007/s11606-0163833-9
12. Penn M, Bhatnagar S, Kuy S, et al. Comparison of wait times for new patients between the private sector and United States Department of Veterans Affairs medical centers. JAMA Netw Open. 2019;2(1):e187096. doi:https://doi.org/10.1001/jamanetworkopen.2018.7096

13. Wright SM, Craig T, Campbell S, Schaefer J, Humble C. Patient satisfaction of female and male users of Veterans Health Administration services. J Gen Intern Med. 2006;21(Suppl 3):S26-S32. doi:https://doi. org/10.1111/j.1525-1497.2006.00371.x

14. Scholle SH, Vuong O, Ding L, et al. Development of and field test results for the CAHPS PCMH Survey. Med Care. 2012;50 Suppl:S2-10. doi:https://doi.org/10.1097/MLR.0b013e3182610aba

15. van Buuren S. Multiple imputation of discrete and continuous data by fully conditional specification. Stat Methods Med Res. 2007;16(3):219242. doi:https://doi.org/10.1177/0962280206074463

16. White I, Royston P, Wood A. Multiple Imputation Using Chained Equations: Issues and Guidance for Practice. Statistics in medicine. https://onlinelibrary.wiley.com/doi/abs/10.1002/sim.4067. Published November 30, 2010.

17. U.S. Department of Veterans Affairs. 172VA10P2: VHA Corporate Data Warehouse - VA. 79 FR 4377. September 2014

18. Elixhauser A, Steiner C, Harris DR, Coffey RM. Comorbidity measures for use with administrative data. Med Care. 1998;36(1):8-27.

19. Rodriguez HP, Knox M, Hurley V, Rittenhouse DR, Shortell SM. The use of enhanced appointment access strategies by medical practices. Med Care. 2016;54(6):632-638. doi:https://doi.org/10.1097/MLR. 0000000000000527

20. Aysola J, Rhodes KV, Polsky D. Patient-centered medical homes and access to services for new primary care patients. Med Care. 2015;53(10):857-62. doi:https://doi.org/10.1097/MLR. 0000000000000412

21. Ghorob A, Bodenheimer T. Sharing the care to improve access to primary care. N Engl J Med. 2012;366(21):1955-1957. doi:https://doi. org/10.1056/NEJMp1202775

22. Nelson KM, Helfrich $\mathbf{C}$, Sun $\mathbf{H}$, et al. Implementation of the patientcentered medical home in the Veterans Health Administration: associations with patient satisfaction, quality of care, staff burnout, and hospital and emergency department use. JAMA Intern Med. 2014;174(8):1350. doi:https://doi.org/10.1001/jamainternmed.2014.2488

23. Reddy A, Pollack CE, Asch DA, Canamucio A, Werner RM. The effect of primary care provider turnover on patient experience of care and ambulatory quality of care. JAMA Intern Med. 2015;175(7):1157-1162. doi:https://doi.org/10.1001/jamainternmed.2015.1853

24. Shipman SA, Sinsky CA. Expanding primary care capacity by reducing waste and improving the efficiency of care. Health Aff (Millwood). 2013;32(11):1990-1997. doi:https://doi.org/10.1377/ hlthaff.2013.0539

25. Sylling PW, Wong ES, Liu C-F, et al. Patient-centered medical home implementation and primary care provider turnover. Med Care. 2014;52(12):1017-1022. doi:https://doi.org/10.1097/MLR. 0000000000000230

26. Helfrich CD, Simonetti JA, Clinton WL, et al. The association of teamspecific workload and staffing with odds of burnout among VA primary care team members. J Gen Intern Med. 2017;32(7):760-766. doi:https:// doi.org/10.1007/s11606-017-4011-4

27. Solimeo SL, Stewart GL, Rosenthal GE. The critical role of clerks in the patient-centered medical home. Ann Fam Med. 2016;14(4):377-379. doi:https://doi.org/10.1370/afm. 1934

28. Burrows M, Gale N, Greenfield S, Litchfield I. Exploring the clinically orientated roles of the general practice receptionist: a systematic review protocol. Syst Rev. 2017;6. doi:https://doi.org/10.1186/s13643-0170612-6

29. Schall MW, Duffy T, Krishnamurthy A, et al. Improving patient access to the Veterans Health Administration's primary care and specialty clinics. Jt Comm J Qual Saf. 2004;30(8):415-423. doi:https://doi.org/10.1016/ S1549-3741(04)30047-X

30. Chesluk BJ, Holmboe ES. How teams work-or don't—in primary care: a field study on internal medicine practices. Health Aff (Millwood). 2010;29(5):874-879. doi:https://doi.org/10.1377/hlthaff.2009.1093

31. Yano EM, Haskell S, Hayes P. Delivery of gender-sensitive comprehensive primary care to women veterans: implications for VA Patient Aligned Care Teams. J Gen Intern Med. 2014;29 Suppl 2:S703-707. doi:https:// doi.org/10.1007/s11606-013-2699-3

32. National Center for Veterans Analysis and Statistics. VA Utilization Profile FY 2016. November 2017. https://www.va.gov/vetdata/docs/ Quickfacts/VA_Utilization_Profile.pdf. . 
33. U.S. Department of Veterans Affairs. Department of Veterans Affairs FY 2018-2024 strategic plan. https://www.va.gov/oei/docs/VA20182024strategicPlan.pdf. Accessed November 27, 2019.

34. U.S. Department of Health \& Human Services. Access to Health Services Heal 2020 Top Object. https://www.healthypeople.gov/2020/topics-objectives/topic/Access-to-Health-Services.

35. Wong ES, Schuttner L, Reddy A. Does machine learning improve prediction of VA primary care reliance? Am J Manag Care. In press 2019

36. Isakson J. VA MISSION Act of 2018.; 2018. https://www.congress.gov/ bill/115th-congress/senate-bill/2372/text.

37. Reddy A, Wong E, Canamucio A, et al. Association between continuity and team-based care and health care utilization: an observational study of Medicare-eligible Veterans in VA patient aligned care team. Health Serv
Res. 2018;53 Suppl 3:5201-5218. doi:https://doi.org/10.1111/14756773.13042

38. Shanafelt T, Goh J, Sinsky C. The business case for investing in physician well-being. JAMA Intern Med. 2017;177(12):1826. doi:https:// doi.org/10.1001/jamainternmed.2017.4340

39. Subramanian U, Ackermann RT, Brizendine EJ, et al. Effect of advanced access scheduling on processes and intermediate outcomes of diabetes care and utilization. J Gen Intern Med. 2009;24(3):327-333. doi:https://doi.org/10.1007/s11606-008-0888-2

Publisher's Note Springer Nature remains neutral with regard to jurisdictional claims in published maps and institutional affiliations. 